This item was submitted to Loughborough's Research Repository by the author.

Items in Figshare are protected by copyright, with all rights reserved, unless otherwise indicated.

\title{
Invariant-geometry conditions for the rational bi-quadratic Bézier surfaces
}

PLEASE CITE THE PUBLISHED VERSION

http://dx.doi.org/10.1016/j.cagd.2009.06.004

PUBLISHER

(C) Elsevier

VERSION

SMUR (Submitted Manuscript Under Review)

LICENCE

CC BY-NC-ND 4.0

REPOSITORY RECORD

Bez, Helmut E.. 2019. "Invariant-geometry Conditions for the Rational Bi-quadratic Bézier Surfaces". figshare. https://hdl.handle.net/2134/14696. 
This item was submitted to Loughborough's Institutional Repository (https://dspace.lboro.ac.uk/) by the author and is made available under the following Creative Commons Licence conditions.

\section{creative
commons}

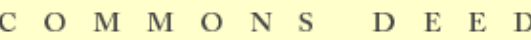

Attribution-NonCommercial-NoDerivs 2.5

You are free:

- to copy, distribute, display, and perform the work

Under the following conditions:

Attribution. You must attribute the work in the manner specified b the author or licensor.

Noncommercial. You may not use this work for commercial purposes.

No Derivative Works. You may not alter, transform, or build upon this work.

- For any reuse or distribution, you must make clear to others the license terms of this work.

- Any of these conditions can be waived if you get permission from the copyright holder.

Your fair use and other rights are in no way affected by the above.

This is a human-readable summary of the Leqal Code (the full license).

\section{Disclaimer 만}

For the full text of this licence, please go to: http://creativecommons.org/licenses/by-nc-nd/2.5/ 


\title{
Invariant-geometry conditions for the rational bi-quadratic Bézier surfaces
}

\author{
H.E. Bez \\ Department of Computer Science, Loughborough University, Loughborough, \\ Leicestershire LE11 3TU. \\ e-mail:h.e.bez@lboro.ac.uk
}

\begin{abstract}
A generalization of Patterson's work (Patterson, 1985), on the invariants of the rational Bézier curves, to the case of surfaces is presented. An equation for the determination of the invariants for surfaces of degree $(n, n)$ is derived and solved for the bi-quadratics - for which it is shown that seven independent, invariant functions exist. Explicit forms of the invariants are derived and a number of applications are presented.
\end{abstract}

\section{Introduction and scope}

Arbitrary transformations of the weights, $\omega_{i}$, of a Bézier curve are geometry modifying; but transformations satisfying certain known conditions preserve the geometry and are equivalent to a re-parametrisation of the curve by Möbius function. In particular Patterson showed that if the weight vector $\omega=\left(\omega_{0}, \ldots, \omega_{n}\right)$ of a degree $n$ Bézier rational path is transformed to $\omega^{*}=\left(\omega_{0}^{*}, \ldots, \omega_{n}^{*}\right)$, where $\omega_{i}>0$ and $\omega_{i}^{*}>0$, then the shape of the path is unchanged if:

$$
\varsigma_{i}\left(\omega^{*}\right)=\varsigma_{i}(\omega) \text { for all } 1 \leq i \leq n-1
$$

where $\varsigma_{i}(\omega)=\frac{\omega_{i-1} \omega_{i+1}}{\omega_{i}^{2}}$. The invariance of the $n-1$ functions $\varsigma_{i}$ therefore provides invariant-geometry conditions for Bézier curves. Additionally the invariants $\varsigma_{i}$ provide information about the curve type and enable normalized weights to be computed (Farin, 1999). The invariants derived in this paper play similar roles for the bi-quadratic Bézier surfaces.

Bézier representations have special properties in the case of all positive weights; for this reason negative weights are often not considered. However in this paper the only restriction on weight values is that they should be non-zero. Negative weights are allowed here for a number of reasons including: 
- completeness and generality

- the difficulty of avoiding negative weights when attempting to parametrise some surfaces, e.g., Dupin cyclides, with a small number of low-degree patches.

Functional and tensor equations for invariant geometry are derived for the rational Bézier surfaces, of degree $(n, n)$. The general solution of the equations is obtained in bi-quadratic case. It is shown that 6 independent, real-valued invariant functions, denoted $\varpi_{1}, \ldots, \varpi_{6}$, and a sign-pattern invariant, denoted, $s p$, exist for these surfaces; and that if the weight vector $\omega$ of a bi-quadratic surface is transformed to $\omega^{*}$ then the shape of the patch is unchanged if and only if:

$$
\varpi_{i}\left(\omega^{*}\right)=\varpi_{i}(\omega) \text {, for } 1 \leq i \leq 6, \text { and } \quad s p\left(\omega^{*}\right)=s p(\omega) .
$$

Explicit forms for the $\varpi$ invariants are derived and some applications are discussed.

\section{Mathematical preliminaries}

\subsection{Functional independence}

If $f_{i}: \mathbb{R}^{q} \rightarrow \mathbb{R}$, for $1 \leq i \leq k$, then the functions $f \equiv\left(f_{1}, \ldots, f_{k}\right)$ are functionally dependent if:

$$
F \circ f=0
$$

for some $F: \mathbb{R}^{k} \rightarrow \mathbb{R}$ not identically zero. The functions are independent if no such $F$ exists. Equivalently, for dependence, we have $(d F \circ f) d f=0$ or, taking the transpose, the linear system:

$$
\left[\begin{array}{cccc}
\partial_{1} f_{1} & \partial_{1} f_{2} & \ldots & \partial_{1} f_{k} \\
\partial_{2} f_{1} & \partial_{2} f_{2} & \ldots & \partial_{2} f_{k} \\
\vdots & \vdots & \vdots & \vdots \\
\partial_{q} f_{1} & \partial_{q} f_{2} & \ldots & \partial_{q} f_{k}
\end{array}\right]\left[\begin{array}{c}
\partial_{1} F \circ f \\
\partial_{2} F \circ f \\
\vdots \\
\partial_{k} F \circ f
\end{array}\right]=0
$$

where $\partial_{i}$ denotes partial derivative with respect to the $i$ th variable. It follows that if $d f$ is of maximal rank, then the only solution is $F \equiv 0$ and the functions $f_{1}, \ldots, f_{k}$ are independent.

In this paper we say that the functions $f_{1}, \ldots, f_{k}$ are manifestly independent if either: 
(1) each $f_{i}$ is a function of a variable that does not occur in the other functions, or

(2) all except one of $f_{1}, \ldots, f_{k}$, is a function of a variable that does not occur in the other functions, and the exceptional function does not depend on any of the variables unique to the others.

For example, the pair $f_{i}: \mathbb{R}^{3} \rightarrow \mathbb{R}$, for $i=1,2$, defined by:

$$
f_{1}(x, y, z)=x y, \quad f_{2}(x, y, z)=y z
$$

is manifestly independent as, respectively, they have variables $x$ and $z$ that do not occur in the other function. It follows that no $F$ with $F\left(f_{1}, f_{2}\right)=0$ can exist. Further, the triple $\left(f_{1}, f_{2}, f_{3}\right)$, where $f_{3}(x, y, z)=y$, is manifestly independent - as $f_{3}$ is not a function of either $x$ or $z$ it cannot be expressed as a function of $f_{1}$ and $f_{2}$. In cases of manifest independence it is not necessary to determine the rank of $d f$.

\subsection{General observations on invariants}

No unique set of invariants exists for a given problem, since further invariants may always be determined as functions of a known set; i.e., if $I_{1}, \ldots, I_{k}$ are real-valued invariants and $h$ is a real-valued function of $k$ variables then $h\left(I_{1}, \ldots, I_{k}\right)$ is an invariant. The invariant problem is therefore one of determining a set of functionally independent invariants that is complete - in the sense that all invariants not in the set may be expressed as a function of those that are. The cardinality of two complete independent sets is the same (Olver, 1999).

A computational approach that produces, directly, a manifestly independent set is of considerable benefit - enabling, for example, the cardinality of complete independent sets to be established immediately. This is important in cases where little is known, apriori, about the invariants sought.The methods used in this paper produce, directly, manifestly functionally independent invariants.

\subsection{Sign-pattern}

Let $A=\{+,-\}$ and $A^{*}$ be the set of all vectors of symbols from $A$. For $\alpha \in A^{*}$ we define $\alpha^{\prime}$ to be the complement of $\alpha$-i.e., the vector obtained by replacing the +'s of $\alpha$ by -'s and the -'s by +'s. We say that two elements, $\alpha, \beta \in A^{*}$ are equivalent (written $\alpha \sim \beta$ ), or have the same sign-pattern, if $\alpha=\beta$ or $\alpha=\beta^{\prime}$. For example $(+,+,-,+,-) \sim(-,-,+,-,+)$. 
The sign-pattern of a vector $X=\left(x_{1}, \ldots, x_{m}\right) \in \mathbb{R}^{m}$, with $x_{i} \neq 0$, may defined to be $\operatorname{sp}(X)=\left[\left(\operatorname{sign}\left(x_{1}\right), \ldots, \operatorname{sign}\left(x_{m}\right)\right)\right] \in A^{*} / \sim$.

\subsection{Equivalent surface parametrisations}

We denote by $\mathcal{S}$, the set of, suitably defined, regular functions $\sigma: I_{1} \times I_{2} \rightarrow$ $\mathbb{R}^{3}$, where $I_{1}$ and $I_{2}$ are intervals of $\mathbb{R}$, that provide local parametrisations of surfaces embedded in $\mathbb{R}^{3}$. The general conditions under which functions parametrise the same surface are as follows: functions $\sigma_{1}, \sigma_{2}$ on, respectively, $I_{1} \times I_{2}$ and $I_{1}^{*} \times I_{2}^{*}$ parametrise the same surface if there is a sufficiently smooth invertible function $\psi: I_{1} \times I_{2} \rightarrow I_{1}^{*} \times I_{2}^{*}$ such that $\sigma_{1}=\sigma_{2} \circ \psi$.

\section{An invariant-geometry functional equation for the Bézier sur- faces of degree $(n, n)$}

If $b_{i}(t)=\left(\begin{array}{l}n \\ i\end{array}\right) t^{i}(1-t)^{n-i}$ then the rational Bernstein surfaces of degree $(n, n)$ take the form

$$
\sigma[v, \omega](t, s)=\frac{\sum_{i, j=0}^{n} b_{i}(t) b_{j}(s) v_{i j}^{*}}{\sum_{i, j=0}^{n} b_{i}(t) b_{j}(s) \omega_{i j}} .
$$

on a set $v=\left\{v_{i j}^{*}: 0 \leq i, j \leq n\right\}$ of $(n+1)^{2}$ vectors and a vector $\omega=$ $\left(\omega_{00}, \omega_{01}, \ldots, \omega_{0 n} ; \omega_{10}, \omega_{11}, \ldots, \omega_{1, n} ; \ldots ; \omega_{n 0}, \ldots \omega_{n n}\right)$ of $(n+1)^{2}$ 'weights'. Rational Bézier surfaces of degree $(n, n)$ are defined for the subset of the Bernstein surfaces for which all the weights are non-zero. In this case the vectors $v_{i j}=\frac{v_{i j}^{*}}{\omega_{i j}}$ are all well-defined and the surface may be written in the Bézier form:

We have

$$
\sigma_{B}[v, \omega](t, s)=\frac{\sum_{i, j=0}^{n} b_{i}(t) b_{j}(s) \omega_{i j} v_{i j}}{\sum_{i, j=0}^{n} b_{i}(t) b_{j}(s) \omega_{i j}}
$$

$$
\sigma_{B}: V \times \Omega \rightarrow \mathcal{S}
$$

where $V$ is the set of all $(n+1)^{2}$-tuples of vectors in $\mathbb{R}^{3}$ and $\Omega$ is the set of all $(n+1)^{2}$-tuples of non-zero real numbers.

Following a little algebra the Bézier surface function $\sigma_{B}[v, \omega]$ may be written in the tensor-product form:

$$
\sigma_{B}[v, \omega](t, s)=\frac{\mathrm{t} \otimes \mathrm{s}\left(B_{n} \otimes B_{n}\right) \Omega_{n} v}{\mathrm{t} \otimes \mathrm{s}\left(B_{n} \otimes B_{n}\right) \omega}
$$

where: 
- $\mathrm{t} \otimes \mathrm{s} \equiv\left[1, t, \ldots, t^{n}\right] \otimes\left[1, s, \ldots, s^{n}\right]$ is the tensor-product basis of the space of polynomials of degree $n$ in two variables; $\mathrm{t} \otimes \mathrm{s}$ is a $1 \times(n+1)^{2}$ vector of polynomial functions which, for $n=2$ is $\left[1, s, s^{2}, t, t s, t s^{2}, t^{2}, t^{2} s, t^{2} s^{2}\right]$

- $B_{n}$ is the lower triangular change-of-basis matrix defined by:

$$
\left[1, t, \ldots, t^{n}\right] B_{n}=\left[b_{0}(t), b_{1}(t), \ldots, b_{n}(t)\right]
$$

and $B_{n} \otimes B_{n}$ is the $(n+1)^{2} \times(n+1)^{2}$ tensor product matrix of $B_{n}$ with $B_{n}$ - $\Omega_{n}$ is the $(n+1)^{2}$ invertible diagonal matrix:

$$
\Omega_{n}=\operatorname{diag}\left(\omega_{00}, \omega_{01}, \ldots, \omega_{0 n} ; \omega_{10}, \omega_{11}, \ldots, \omega_{1, n} ; \ldots ; \omega_{n 0}, \ldots \omega_{n n}\right)
$$

- $v=\left(v_{00}, \ldots, v_{0 n} ; v_{10}, \ldots, v_{1 n} ; \ldots ; v_{n 0}, \ldots, v_{n n}\right)^{T}$

- $\omega=\left(\omega_{00}, \omega_{01}, \ldots, \omega_{0 n} ; \omega_{10}, \omega_{11}, \ldots, \omega_{1, n} ; \ldots ; \omega_{n 0}, \ldots, \omega_{n n}\right)^{T}$.

From the general conditions of section 2.4, it follows that weight vectors $\omega, \omega^{*} \in \Omega$ determine surfaces with the same geometry, for all $v \in V$, if and only if there exists a re-parametrisation function $\psi$ such that

$$
\sigma_{B}[v, \omega]=\sigma_{B}\left[v, \omega^{*}\right] \circ \psi \text { for all } v \in V .
$$

The invariant-geometry conditions for Bézier surfaces of degree $(n, n)$ may be obtained from the general solution of this functional equation - comprising $\psi$ solutions for all weight vector pairs $\omega, \omega^{*}$.

As re-parametrisation functions $\psi$ may be written in the paired form

$$
\psi(t, s)=(\phi(t, s), \mu(t, s))
$$

the function $\sigma_{B}[v, \omega] \circ \psi$ may be expressed as

$$
\sigma_{B}[v, \omega] \circ \psi=\frac{\phi \otimes \mu\left(B_{n} \otimes B_{n}\right) \Omega_{n} v}{\phi \otimes \mu\left(B_{n} \otimes B_{n}\right) \omega},
$$

where $\phi \otimes \mu=\left[1, \ldots, \phi^{n}\right] \otimes\left[1, \ldots, \mu^{n}\right]$. The invariant-geometry functional equation for Bézier surfaces, of degree $(n, n)$ may therefore be written:

$$
\frac{\phi \otimes \mu\left(B_{n} \otimes B_{n}\right) \Omega_{n}^{*} v}{\phi \otimes \mu\left(B_{n} \otimes B_{n}\right) \omega^{*}}=\frac{\mathrm{t} \otimes \mathrm{s}\left(B_{n} \otimes B_{n}\right) \Omega_{n} v}{\mathrm{t} \otimes \mathrm{s}\left(B_{n} \otimes B_{n}\right) \omega}
$$

where $\Omega_{n}^{*}$ is the $(n+1)^{2} \times(n+1)^{2}$ diagonal matrix defined by:

$$
\Omega_{n}^{*}=\operatorname{diag}\left(\omega_{00}^{*}, \omega_{01}^{*}, \ldots, \omega_{0 n}^{*} ; \omega_{10}^{*}, \omega_{11}^{*}, \ldots, \omega_{1, n}^{*} ; \ldots ; \omega_{n 0}^{*}, \ldots, \omega_{n n}^{*}\right) .
$$

and $\omega^{*}$ is the $(n+1)^{2} \times 1$ column vector

$$
\omega^{*}=\left(\omega_{00}^{*}, \omega_{01}^{*}, \ldots, \omega_{0 n}^{*} ; \omega_{10}^{*}, \omega_{11}^{*}, \ldots, \omega_{1, n}^{*} ; \ldots ; \omega_{n 0}^{*}, \ldots, \omega_{n n}^{*}\right)^{T} .
$$


As relation 1 is required to hold for all $v$ it reduces to:

$$
\frac{\phi \otimes \mu\left(B_{n} \otimes B_{n}\right) \Omega_{n}^{*}}{\phi \otimes \mu\left(B_{n} \otimes B_{n}\right) \omega^{*}}=\frac{\mathrm{t} \otimes \mathrm{s}\left(B_{n} \otimes B_{n}\right) \Omega_{n}}{\mathrm{t} \otimes \mathrm{s}\left(B_{n} \otimes B_{n}\right) \omega} .
$$

\section{The bi-quadratic case}

\subsection{Functional solutions for bi-quadratic surfaces}

In the remainder of the paper $\sigma_{B}$ denotes the rational bi-quadratic Bézier surface function. The ratios $\frac{\omega_{i j}}{\omega_{i j}^{*}}$ occur naturally in the solution of equation 2 , and are denoted henceforth by $\rho_{i j}$. For the bi-quadratics equation 2 is:

$$
\frac{\phi \otimes \mu\left(B_{2} \otimes B_{2}\right) \Omega_{2}^{*}}{\phi \otimes \mu\left(B_{2} \otimes B_{2}\right) \omega^{*}}=\frac{\mathrm{t} \otimes \mathrm{s}\left(B_{2} \otimes B_{2}\right) \Omega_{2}}{\mathrm{t} \otimes \mathrm{s}\left(B_{2} \otimes B_{2}\right) \omega} .
$$

where

$$
B_{2} \otimes B_{2}=\left[\begin{array}{ccccccccc}
1 & 0 & 0 & 0 & 0 & 0 & 0 & 0 & 0 \\
-2 & 2 & 0 & 0 & 0 & 0 & 0 & 0 & 0 \\
1 & -2 & 1 & 0 & 0 & 0 & 0 & 0 & 0 \\
-2 & 0 & 0 & 2 & 0 & 0 & 0 & 0 & 0 \\
4 & -4 & 0 & -4 & 4 & 0 & 0 & 0 & 0 \\
-2 & 4 & -2 & 2 & -4 & 2 & 0 & 0 & 0 \\
1 & 0 & 0 & -2 & 0 & 0 & 1 & 0 & 0 \\
-2 & 2 & 0 & 4 & -4 & 0 & -2 & 2 & 0 \\
1 & -2 & 1 & -2 & 4 & -2 & 1 & -2 & 1
\end{array}\right]
$$

and

$$
\Omega_{2}=\operatorname{diag}\left(\omega_{00}, \omega_{01}, \ldots, \omega_{22}\right), \quad \Omega_{2}^{*}=\operatorname{diag}\left(\omega_{00}^{*}, \omega_{01}^{*}, \ldots, \omega_{22}^{*}\right) .
$$

All the $\psi \equiv(\phi, \psi)$ solutions of equation (3) are required; while it is known that pairs of Möbius functions $(\phi(t), \mu(s))=\left(\frac{a t}{1+(a-1) t}, \frac{b s}{1+(b-1) s}\right)$ are solutions, the following Lemma establishes that there are no others.

Lemma 1. If $\psi=(\phi, \mu)$ is invertible and satisfies equation (3), for the weight vectors $\left(\omega, \omega^{*}\right)$, then:

$$
\phi(t, s)=\frac{\rho_{21} t}{\rho_{11}+\left(\rho_{21}-\rho_{11}\right) t}, \quad \mu(t, s)=\frac{\rho_{22} s}{\rho_{21}+\left(\rho_{22}-\rho_{21}\right) s}
$$

and $\rho_{11}, \rho_{21}$ and $\rho_{22}$ are all of the same sign.

Proof. Relation 3 is equivalent to: 
Elements $(1,9)$ and $(1,8)$ of the array on each side of $(4)$, above, give respectively:

$$
\begin{aligned}
\left(\mathrm{t} \otimes \mathrm{s}\left(B_{2} \otimes B_{2}\right) \omega\right) \phi^{2} \mu^{2} \omega_{22}^{*} & =\left(\phi \otimes \mu\left(B_{2} \otimes B_{2}\right) \omega^{*}\right) t^{2} s^{2} \omega_{22} \\
\left(\mathrm{t} \otimes \mathrm{s}\left(B_{2} \otimes B_{2}\right) \omega\right)\left[\phi^{2} \mu-\phi^{2} \mu^{2}\right] \omega_{21}^{*} & =\left(\phi \otimes \mu\left(B_{2} \otimes B_{2}\right) \omega^{*}\right)\left[t^{2} s-t^{2} s^{2}\right] \omega_{21}
\end{aligned}
$$

which are easily solved for $\mu$, by considering the ratio $(5) /(6)$, to give:

$$
\mu(s)=\frac{\rho_{22} s}{\rho_{21}+\left(\rho_{22}-\rho_{21}\right) s} .
$$

The $(1,5)$ components of equation 4 give:

$\left(\mathrm{t} \otimes \mathrm{s}\left(B_{2} \otimes B_{2}\right) \omega\right)\left[\phi \mu-\phi \mu^{2}-\phi^{2} \mu+\phi^{2} \mu^{2}\right] \omega_{11}^{*}=\left(\phi \otimes \mu\left(B_{2} \otimes B_{2}\right) \omega^{*}\right)\left[t s-t s^{2}-t^{2} s+t^{2} s^{2}\right] \omega_{11}$

which, together with the $(1,8)$ components and the solution for $\mu$, may be solved for $\phi$ as:

$$
\phi(t)=\frac{\rho_{21} t}{\rho_{11}+\left(\rho_{21}-\rho_{11}\right) t}
$$

i.e., the solutions for $\mu$ and $\phi$ are both Möbius functions of a single variable with the constants identified above.

We have

$$
\phi^{\prime}(t)=\frac{\rho_{11} \rho_{21}}{\left(\rho_{11}+\left(\rho_{21}-\rho_{11}\right) t\right)^{2}} \text { and } \mu^{\prime}(t)=\frac{\rho_{22} \rho_{21}}{\left(\rho_{21}+\left(\rho_{22}-\rho_{21}\right) s\right)^{2}} .
$$

Invertbility of $\psi$ is equivalent to non-vanishing Jacobian on $[0,1] \times[0,1]$. We have:

$$
J \psi(t, s)=\operatorname{det}\left[\begin{array}{cc}
\phi^{\prime}(t) & 0 \\
0 & \mu^{\prime}(s)
\end{array}\right]=\phi^{\prime}(t) \mu^{\prime}(s)
$$

i.e., invertibility requires $\phi^{\prime}(t) \neq 0$ for all $t \in[0,1]$ and $\mu^{\prime}(s) \neq 0$ for all $s \in[0,1]$ - equivalently that $\phi$ and $\mu$ are monotone. Hence $\phi:[0,1] \rightarrow[0,1]$ is strictly increasing - similarly $\mu$. Hence:

- $\rho_{11} \rho_{21}>0$, equivalently $\rho_{11}$ and $\rho_{21}$ have the same sign

- $\rho_{22} \rho_{21}>0$, equivalently $\rho_{21}$ and $\rho_{22}$ have the same sign

i.e., $\rho_{11}, \rho_{21}$ and $\rho_{22}$ all have the same sign.

\subsection{Reduced invariant-geometry conditions}

The following Lemma reduces the invariant-geometry condition (3) to two, equivalent, simpler relations that yield the invariant functions directly. 
Lemma 2. The invariant-geometry condition for the bi-quadratics (3) is equivalent to:

$$
\omega^{*}=c\left(B_{2}^{-1} U_{\phi}^{-1} B_{2}\right) \otimes\left(B_{2}^{-1} U_{\mu}^{-1} B_{2}\right) \omega
$$

or

$$
\Omega_{2}^{*}=c\left(B_{2}^{-1} U_{\phi}^{-1} B_{2}\right) \otimes\left(B_{2}^{-1} U_{\mu}^{-1} B_{2}\right) \Omega_{2}
$$

where $c$ is an arbitrary non-zero multiplier,

$$
U_{\phi}=\left[\begin{array}{ccc}
\rho_{11}^{2} & 0 & 0 \\
2 \rho_{11}\left(\rho_{21}-\rho_{11}\right) & \rho_{11} \rho_{21} & 0 \\
\left(\rho_{21}-\rho_{11}\right)^{2} & \rho_{21}\left(\rho_{21}-\rho_{11}\right) & \rho_{21}^{2}
\end{array}\right]
$$

and

$$
U_{\mu}=\left[\begin{array}{ccc}
\rho_{21}^{2} & 0 & 0 \\
2 \rho_{21}\left(\rho_{22}-\rho_{21}\right) & \rho_{21} \rho_{22} & 0 \\
\left(\rho_{22}-\rho_{21}\right)^{2} & \rho_{22}\left(\rho_{22}-\rho_{21}\right) & \rho_{22}^{2}
\end{array}\right] \text {. }
$$

Proof. As the general solutions to (3) for $\phi$ and $\mu$ are now known, (3) transforms to a tensor relationship in $s, t, \omega$ and $\omega^{*}$ with polynomial elements. Writing

$$
\phi=\frac{\rho_{21} t}{\rho_{11}+\left(\rho_{21}-\rho_{11}\right) t} \equiv \frac{\phi_{N}}{\phi_{D}} \quad \text { and } \quad \mu=\frac{\rho_{22} s}{\rho_{21}+\left(\rho_{22}-\rho_{21}\right) s} \equiv \frac{\mu_{N}}{\mu_{D}}
$$

it is easy to show that:

$$
\left[\phi_{D}^{2}, \phi_{D} \phi_{N}, \phi_{N}^{2}\right]=\left[1, t, t^{2}\right] U_{\phi} \quad \text { and } \quad\left[\mu_{D}^{2}, \mu_{D} \mu_{N}, \mu_{N}^{2}\right]=\left[1, s, s^{2}\right] U_{\mu}
$$

where $U_{\phi}$ and $U_{\mu}$ are specified in the statement of the Proposition. it follows that:

$$
\begin{aligned}
\frac{\left[1, t, t^{2}\right] \otimes\left[1, s, s^{2}\right]\left(B_{2} \otimes B_{2}\right) \Omega_{2}}{\left[1, t, t^{2}\right] \otimes\left[1, s, s^{2}\right]\left(B_{2} \otimes B_{2}\right) \omega} & =\frac{\left[1, \phi, \phi^{2}\right] \otimes\left[1, \mu, \mu^{2}\right]\left(B_{2} \otimes B_{2}\right) \Omega_{2}^{*}}{\left[1, \phi, \phi^{2}\right] \otimes\left[1, \mu, \mu^{2}\right]\left(B_{2} \otimes B_{2}\right) \omega^{*}} \\
& =\frac{\left[\phi_{D}^{2}, \phi_{D} \phi_{N}, \phi_{N}^{2}\right] \otimes\left[\mu_{D}^{2}, \mu_{D} \mu_{N}, \mu_{N}^{2}\right]\left(B_{2} \otimes B_{2}\right) \Omega_{2}^{*}}{\left[\phi_{D}^{2}, \phi_{D} \phi_{N}, \phi_{N}^{2}\right] \otimes\left[\mu_{D}^{2}, \mu_{D} \mu_{N}, \mu_{N}^{2}\right] \omega^{*}} \\
& =\frac{\left[1, t, t^{2}\right] \otimes\left[1, s, s^{2}\right]\left(U_{\phi} \otimes U_{\mu}\right)\left(B_{2} \otimes B_{2}\right) \Omega_{2}^{*}}{\left[1, t, t^{2}\right] \otimes\left[1, s, s^{2}\right]\left(U_{\phi} \otimes U_{\mu}\right)\left(B_{2} \otimes B_{2}\right) \omega^{*}} .
\end{aligned}
$$


As the above is required to hold for all $(t, s)$ it follows that:

$$
\frac{\left(B_{2} \otimes B_{2}\right) \Omega_{2}}{\left(B_{2} \otimes B_{2}\right) \omega}=\frac{\left(U_{\phi} \otimes U_{\mu}\right)\left(B_{2} \otimes B_{2}\right) \Omega_{2}^{*}}{\left(U_{\phi} \otimes U_{\mu}\right)\left(B_{2} \otimes B_{2}\right) \omega^{*}} .
$$

Hence for some arbitrary non-zero multiplier $c$ we have:

$$
\left(U_{\phi} \otimes U_{\mu}\right)\left(B_{2} \otimes B_{2}\right) \omega^{*}=c\left(B_{2} \otimes B_{2}\right) \omega \text { and } \quad\left(U_{\phi} \otimes U_{\mu}\right)\left(B_{2} \otimes B_{2}\right) \Omega_{2}^{*}=c\left(B_{2} \otimes B_{2}\right) \Omega_{2},
$$

or

$$
\begin{aligned}
& \omega^{*}=c\left(B_{2}^{-1} U_{\phi}^{-1} B_{2}\right) \otimes\left(B_{2}^{-1} U_{\mu}^{-1} B_{2}\right) \omega \\
& \Omega_{2}^{*}=c\left(B_{2}^{-1} U_{\phi}^{-1} B_{2}\right) \otimes\left(B_{2}^{-1} U_{\mu}^{-1} B_{2}\right) \Omega_{2}
\end{aligned}
$$

The equivalence of these two relations follows from the diagonal nature of $B_{2}^{-1} U_{\phi}^{-1} B_{2}$ and $B_{2}^{-1} U_{\mu}^{-1} B_{2}$. We have

$B_{2}^{-1} U_{\phi}^{-1} B_{2}=\operatorname{diag}\left(\frac{1}{\rho_{11}^{2}}, \frac{1}{\rho_{11} \rho_{21}}, \frac{1}{\rho_{21}^{2}}\right)$ and $B_{2}^{-1} U_{\mu}^{-1} B_{2}=\operatorname{diag}\left(\frac{1}{\rho_{21}^{2}}, \frac{1}{\rho_{21} \rho_{22}}, \frac{1}{\rho_{22}{ }^{2}}\right)$

hence $\left(B_{2}^{-1} U_{\phi}^{-1} B_{2}\right) \otimes\left(B_{2}^{-1} U_{\mu}^{-1} B_{2}\right)$ is diagonal. Trivially, if $A$ is a diagonal matrix and $x$ a column vector then $x^{*}=A x$ if and only if $\operatorname{diag}\left(x^{*}\right)=A \operatorname{diag}(x)$; hence (7) if and only if (8) and the proof is complete.

\subsection{Invariant-geometry transformations and the invariant functions}

From Lemma 2, (7) is a necessary and sufficient condition for invariant geometry - similarly (8). Proposition 1 shows that (7) is a generalization, to the case of mixed-sign weight vectors being allowed, of a known constant-geometry, weight-transformation rule. Relation (8) leads directly to the determination of the invariant functions of the transformations determined by (7) - see Proposition 2 .

Proposition 1. The invariant-geometry condition (7) is equivalent to the following relationship between $\omega$ and $\omega^{*}$ :

$$
\omega^{*}= \pm e^{\alpha} \operatorname{diag}\left(1, e^{\beta}, e^{2 \beta}, e^{\gamma}, e^{\gamma+\beta}, e^{\gamma+2 \beta}, e^{2 \gamma}, e^{2 \gamma+\beta}, e^{2 \gamma+2 \beta}\right) \omega
$$

for $\alpha, \beta, \gamma \in \mathbb{R}$.

Proof. From the proof of Lemma 2 we have: 


$$
\begin{aligned}
& \left(B_{2}^{-1} U_{\phi}^{-1} B_{2}\right) \otimes\left(B_{2}^{-1} U_{\mu}^{-1} B_{2}\right)= \\
& \operatorname{diag}\left(\frac{1}{\rho_{11}^{2} \rho_{21}^{2}}, \frac{1}{\rho_{11}^{2} \rho_{21} \rho_{22}}, \frac{1}{\rho_{11}^{2} \rho_{22} 2}, \frac{1}{\rho_{11} \rho_{21}^{3}}, \frac{1}{\rho_{11} \rho_{21}^{2} \rho_{22}}, \frac{1}{\rho_{11} \rho_{21} \rho_{22}^{2}}, \frac{1}{\rho_{21} 4}, \frac{1}{\rho_{21}{ }^{3} \rho_{22}}, \frac{1}{\rho_{21}{ }^{2} \rho_{22}^{2}}\right) .
\end{aligned}
$$

Since $\rho_{11}, \rho_{21}$ and $\rho_{22}$ are all of the same sign (Lemma 1), the elements of the matrix $\left(B_{2}^{-1} U_{\phi}^{-1} B_{2}\right) \otimes\left(B_{2}^{-1} U_{\mu}^{-1} B_{2}\right)$ are all positive. We may therefore define $\alpha, \beta$ and $\gamma$ by:

$$
e^{\alpha}=\frac{|c|}{\rho_{11}^{2} \rho_{21}^{2}} \quad e^{\beta}=\frac{\rho_{21}}{\rho_{22}}, \quad e^{\gamma}=\frac{\rho_{11}}{\rho_{21}},
$$

and re-write invariant-geometry condition (7), as:

$$
\omega^{*}= \pm e^{\alpha} \operatorname{diag}\left(1, e^{\beta}, e^{2 \beta}, e^{\gamma}, e^{\gamma+\beta}, e^{\gamma+2 \beta}, e^{2 \gamma}, e^{2 \gamma+\beta}, e^{2 \gamma+2 \beta}\right) \omega .
$$

The transformations (11) of Proposition 1 determine a 3-parameter subgroup of the general linear group $G L(9, \mathbb{R})$. They may be written as $\omega_{i j}^{*}= \pm e^{\alpha}\left(e^{\gamma}\right)^{i}\left(e^{\beta}\right)^{j} \omega_{i j}$ and amount to a generalization of the weight transformations implied by the expression for re-parametrised Bézier surfaces (see Farin (1999) page 194), to the case of mixed-sign weight vectors.

Proposition 2. The invariant-geometry condition (8) is equivalent to the following on $\omega$ and $\omega^{*}$ :

(1) $\operatorname{sp}\left(\omega^{*}\right)=\operatorname{sp}(\omega)$

(2) $\varpi_{i}\left(\omega^{*}\right)=\varpi_{i}(\omega)$ for $1 \leq i \leq 6$

where

$$
\begin{aligned}
& \varpi_{1}(\omega)=\frac{\omega_{01}}{\omega_{00}}\left(\frac{\omega_{21}}{\omega_{22}}\right), \varpi_{2}(\omega)=\frac{\omega_{02}}{\omega_{00}}\left(\frac{\omega_{21}^{2}}{\omega_{22}^{2}}\right), \varpi_{3}(\omega)=\frac{\omega_{10}}{\omega_{00}}\left(\frac{\omega_{11}}{\omega_{21}}\right), \\
& \varpi_{4}(\omega)=\frac{\omega_{11}}{\omega_{00}}\left(\frac{\omega_{11}}{\omega_{22}}\right), \varpi_{5}(\omega)=\frac{\omega_{12}}{\omega_{00}}\left(\frac{\omega_{11} \omega_{21}}{\omega_{22}^{2}}\right), \varpi_{6}(\omega)=\frac{\omega_{20}}{\omega_{00}}\left(\frac{\omega_{11}^{2}}{\omega_{21}^{2}}\right) .
\end{aligned}
$$

Proof. We have $\Omega_{2}^{*}=c \quad\left(B_{2}^{-1} U_{\phi}^{-1} B_{2}\right) \otimes\left(B_{2}^{-1} U_{\mu}^{-1} B_{2}\right) \Omega_{2}$ where the matrix $\left(B_{2}^{-1} U_{\phi}^{-1} B_{2}\right) \otimes\left(B_{2}^{-1} U_{\mu}^{-1} B_{2}\right)$ has positive elements (proof of Proposition 1). Given that $c$ can take either sign, it follows that the signs of all the diagonal elements of $\Omega_{2}^{*}$ are the same as the signs of those of $\Omega_{2}$ or are all of opposite sign. Hence $s p\left(\omega^{*}\right)=s p(\omega)$. Re-writing (8) as:

$$
\frac{1}{c} I_{9}=\left(B_{2}^{-1} U_{\phi}^{-1} B_{2}\right) \otimes\left(B_{2}^{-1} U_{\mu}^{-1} B_{2}\right) \Omega_{2} \Omega_{2}^{*-1}
$$

where $I_{9}$ is the $9 \times 9$ identity matrix, and using the explicit form for $\left(B_{2}^{-1} U_{\phi}^{-1} B_{2}\right) \otimes$ $\left(B_{2}^{-1} U_{\mu}^{-1} B_{2}\right)$ and $\Omega_{2} \Omega_{2}^{*-1}=\operatorname{diag}\left(\rho_{00}, \ldots, \rho_{22}\right)$ we obtain, following cancella- 
tions in three terms:

$$
\frac{1}{c} I_{9}=\operatorname{diag}\left(\frac{\rho_{00}}{\rho_{11}^{2} \rho_{21}^{2}}, \frac{\rho_{01}}{\rho_{11}^{2} \rho_{21} \rho_{22}}, \frac{\rho_{02}}{\rho_{11}^{2} \rho_{22}{ }^{2}}, \frac{\rho_{10}}{\rho_{11} \rho_{21}^{3}}, \frac{1}{\rho_{21}^{2} \rho_{22}}, \frac{\rho_{12}}{\rho_{11} \rho_{21} \rho_{22}^{2}}, \frac{\rho_{20}}{\rho_{21}}, \frac{1}{\rho_{21}{ }^{2} \rho_{22}}, \frac{1}{\rho_{21}{ }^{2} \rho_{22}}\right) .
$$

It follows that the elements along the diagonal of the matrix on the right-hand side of the equation are all equal. This gives the equations:

$\frac{\rho_{00}}{\rho_{11}^{2} \rho_{21}^{2}}=\frac{\rho_{01}}{\rho_{11}^{2} \rho_{21} \rho_{22}}=\frac{\rho_{02}}{\rho_{11}^{2} \rho_{22}^{2}}=\frac{\rho_{10}}{\rho_{11} \rho_{21}^{3}}=\frac{1}{\rho_{21}^{2} \rho_{22}}=\frac{\rho_{12}}{\rho_{11} \rho_{21} \rho_{22}^{2}}=\frac{\rho_{20}}{\rho_{21}^{4}}=\frac{1}{\rho_{21}^{2} \rho_{22}}=\frac{1}{\rho_{21}^{2} \rho_{22}}$

which reduce trivially, because the term $\frac{1}{\rho_{21}^{2} \rho_{22}}$ occurs three times, to the 6 equations:

$$
\frac{\rho_{00}}{\rho_{11}^{2} \rho_{21}^{2}}=\frac{\rho_{01}}{\rho_{11}^{2} \rho_{21} \rho_{22}}=\frac{\rho_{02}}{\rho_{11}^{2} \rho_{22}^{2}}=\frac{\rho_{10}}{\rho_{11} \rho_{21}^{3}}=\frac{1}{\rho_{21}^{2} \rho_{22}}=\frac{\rho_{12}}{\rho_{11} \rho_{21} \rho_{22}^{2}}=\frac{\rho_{20}}{\rho_{21}^{4}}
$$

for the 9 unknowns $\rho_{00}, \ldots, \rho_{22}$. The first equation is equivalent to $\frac{\rho_{00} \rho_{22}}{\rho_{21} \rho_{01}}=$ 1 , or $\varpi_{1}\left(\omega^{*}\right)=\varpi_{1}(\omega)$. The remaining equations demonstrate the invariance of $\varpi_{2}, \ldots, \varpi_{6}$ in a similar way. As each step may be reversed the proof is complete.

It should be noted that the conclusion $s p\left(\omega^{*}\right)=s p(\omega)$ may also be drawn directly from (7).

The following are immediate from Propositions 1 and 2 respectively. Corollary 2 is the generalization of Patterson's results for Bézier curves to the bi-quadratic Bézier surfaces.

Corollary 1. Two bi-quadratic Bézier functions with weight vectors $\omega=$ $\left(\omega_{00}, \ldots, \omega_{22}\right)$ and $\omega^{*}=\left(\omega_{00}^{*}, \ldots, \omega_{22}^{*}\right)$ and identical vertex sets $v$, parametrise the same region of a surface on the domain $[0,1] \times[0,1]$, for all $v \in V$, if and only if there exist $\alpha, \beta, \gamma \in \mathbb{R}$ such that:

$$
\omega^{*}= \pm e^{\alpha} \operatorname{diag}\left(1, e^{\beta}, e^{2 \beta}, e^{\gamma}, e^{\gamma+\beta}, e^{\gamma+2 \beta}, e^{2 \gamma}, e^{2 \gamma+\beta}, e^{2 \gamma+2 \beta}\right) \omega .
$$

Corollary 2. Two bi-quadratic Bézier functions with weight vectors $\omega=$ $\left(\omega_{00}, \ldots, \omega_{22}\right)$ and $\omega^{*}=\left(\omega_{00}^{*}, \ldots, \omega_{22}^{*}\right)$ and identical vertex sets $v$, parametrise the same region of a surface on the domain $[0,1] \times[0,1]$, for all $v \in V$, if and only if:

$$
s p\left(\omega^{*}\right)=s p(\omega) \quad \text { and } \quad \varpi_{i}\left(\omega^{*}\right)=\varpi_{i}(\omega) \quad \text { for } 1 \leq i \leq 6 .
$$




\subsection{Properties of the invariants}

From the functional forms of the type $\varpi$ invariants is clear that all except $\varpi_{4}$ is a function of a variable that does not occur in the others. For $\varpi_{1}$ the variable is $\omega_{01}$, for $\varpi_{2}$ it is $\omega_{02}$, for $\varpi_{3}$ it is $\omega_{10}$, for $\varpi_{5}$ it is $\omega_{12}$ and for $\varpi_{6}$ it is $\omega_{20}$; further $\varpi_{4}$ is not a function of these variables. It follows that $\left\{\varpi_{1}, \ldots, \varpi_{6}\right\}$ is a manifestly functionally independent set.

The independence of the sign-pattern invariant, $s p$, from the type $\varpi$ invariants can be demonstrated as follows: define weight vectors $\omega$ and $\omega^{*}$ by

$$
\begin{aligned}
\omega & =\left(\omega_{00}, \omega_{01}, \omega_{02}, \omega_{10}, \omega_{11}, \omega_{12}, \omega_{20}, \omega_{21}, \omega_{22}\right) \\
\omega^{*} & =\left(\omega_{00},-\omega_{01}, \omega_{02},-\omega_{10}, \omega_{11},-\omega_{12}, \omega_{20},-\omega_{21}, \omega_{22}\right) .
\end{aligned}
$$

it is easy to check that $\varpi_{i}\left(\omega^{*}\right)=\varpi_{i}(\omega)$ for $1 \leq i \leq 6$ but, clearly, $\operatorname{sp}\left(\omega^{*}\right) \neq$ $s p(\omega)$. Hence the invariance of $s p$ is not not implied by the invariance of the $\varpi_{i}$ functions. We have:

Proposition 3. (i) The functions $\left\{\varpi_{1}, \ldots, \varpi_{6}, s p\right\}$ constitute a complete, functionally independent set of invariants for the bi-quadratic Bézier surfaces. (ii) All complete, functionally independent sets of invariants for the bi-quadratic Bézier surfaces have 7 elements.

Proof. By Proposition 1 a complete set of invariants has no more than seven elements, but $\left\{\varpi_{1}, \ldots, \varpi_{6}, s p\right\}$ are independent - hence (i). Invariance of cardinality (Olver, 1999) gives (ii).

\subsection{Canonical-form invariants for bi-quadratic surfaces}

In this section a complete, independent set of invariants, bearing a close relationship to Patterson's forms for Bézier curves, is shown to exist for the bi-quadratic Bézier surfaces.

Proposition 4. The sign pattern invariant sp and the functions $\varpi_{1}^{*}, \ldots \varpi_{6}^{*}$, defined by,

$$
\begin{array}{lll}
\varpi_{1}^{*}(\omega)=\frac{\omega_{00} \omega_{20}}{\omega_{10}^{2}}, & \varpi_{2}^{*}(\omega)=\frac{\omega_{01} \omega_{21}}{\omega_{11}^{2}}, & \varpi_{3}^{*}(\omega)=\frac{\omega_{02} \omega_{22}}{\omega_{12}^{2}}, \\
\varpi_{4}^{*}(\omega)=\frac{\omega_{00} \omega_{02}}{\omega_{01}^{2}}, & \varpi_{5}^{*}(\omega)=\frac{\omega_{10} \omega_{12}}{\omega_{11}^{2}}, & \varpi_{6}^{*}(\omega)=\frac{\omega_{20} \omega_{22}}{\omega_{21}^{2}},
\end{array}
$$

comprise an alternative complete set of functionally independent invariants for the bi-quadratic Bézier surfaces.

Proof. The properties of invariance and independence need to be established for the functions $\left\{\varpi_{1}^{*}, \ldots, \varpi_{6}^{*}\right\}$. 
(i) As shown earlier, the invariant-geometry transformations are:

$$
g_{(\alpha, \beta, \gamma)}= \pm e^{\alpha} \operatorname{diag}\left(1, e^{\beta}, e^{2 \beta}, e^{\gamma}, e^{\gamma+\beta}, e^{\gamma+2 \beta}, e^{2 \gamma}, e^{2 \gamma+\beta}, e^{2 \gamma+2 \beta}\right) .
$$

It follows that $\varpi_{1}^{*}$ transforms as:

$$
\begin{aligned}
\varpi_{1}^{*}\left(g_{(\alpha, \beta, \gamma)} \omega\right) & =\frac{\left( \pm e^{\alpha}\right) \omega_{00}\left( \pm e^{\alpha}\right) e^{2 \gamma} \omega_{20}}{\left( \pm e^{\alpha}\right)^{2}\left(e^{\gamma}\right)^{2} \omega_{10}^{2}} \\
& =\frac{\omega_{00} \omega_{20}}{\omega_{10}^{2}} \\
& =\varpi_{1}^{*}(\omega) .
\end{aligned}
$$

Similar arguments hold for $\varpi_{2}^{*}, \ldots, \varpi_{6}^{*}$ to complete the proof of invariance.

(ii) The type $\varpi^{*}$ invariants are not manifestly independent; the rank of the matrix $d \varpi^{*}$ should therefore be determined. We have

$$
d \varpi^{*}(\omega)=\left[\begin{array}{cccccc}
\frac{\omega_{02}}{\omega_{10}^{2}} & 0 & 0 & \frac{\omega_{02}}{\omega_{01}^{2}} & 0 & 0 \\
0 & \frac{\omega_{21}}{\omega_{11}^{2}} & 0 & -2 \frac{\omega_{00} \omega_{02}}{\omega_{01}^{3}} & 0 & 0 \\
0 & 0 & \frac{\omega_{22}}{\omega_{12}^{2}} & \frac{\omega_{00}}{\omega_{01}^{2}} & 0 & 0 \\
-2 \frac{\omega_{00} \omega_{20}}{\omega_{10}^{3}} & 0 & 0 & 0 & \frac{\omega_{12}}{\omega_{11}^{2}} & 0 \\
0 & -2 \frac{\omega_{01} \omega_{21}}{\omega_{11}^{3}} & 0 & 0 & -2 \frac{\omega_{11} \omega_{12}}{\omega_{11}^{3}} & 0 \\
0 & 0 & -2 \frac{\omega_{02} \omega_{22}}{\omega_{12}^{3}} & 0 & \frac{\omega_{10}}{\omega_{11}^{2}} & 0 \\
\frac{\omega_{00}}{\omega_{10}^{2}} & 0 & 0 & 0 & 0 & \frac{\omega_{22}}{\omega_{21}^{2}} \\
0 & \frac{\omega_{01}}{\omega_{11}^{2}} & 0 & 0 & 0 & -2 \frac{\omega_{20} \omega_{22}}{\omega_{21}^{3}} \\
0 & 0 & \frac{\omega_{02}}{\omega_{12}^{2}} & 0 & 0 & \frac{\omega_{20}}{\omega_{21}^{2}}
\end{array}\right]
$$

which is of full rank (i.e., 6). Hence $\varpi_{1}^{*}, \ldots, \varpi_{6}^{*}$ are functionally independent. Finally the weight vectors:

$$
\begin{aligned}
& \omega=\left(\omega_{00}, \omega_{01}, \omega_{02}, \omega_{10}, \omega_{11}, \omega_{12}, \omega_{20}, \omega_{21}, \omega_{22}\right) \\
& \omega^{*}=\left(-\omega_{00}, \omega_{01},-\omega_{02},-\omega_{10}, \omega_{11},-\omega_{12},-\omega_{20},-\omega_{21},-\omega_{22}\right)
\end{aligned}
$$

are such that $\varpi_{i}^{*}\left(\omega^{*}\right)=\varpi_{i}^{*}(\omega)$ but $s p\left(\omega^{*}\right) \neq s p(\omega)$. Hence the invariance of $s p$ is not implied by the type $\varpi^{*}$ invariants.

The type $\varpi$ and type $\varpi^{*}$ invariants have complementary properties. The canonical-form invariants have a clear geometric relationship with quadratic curves in the surface - they correspond to curves along the vertical and horizontal straight lines in Figure 1. The manifestly-independent invariants lead rapidly to conclusions concerning analytic issues - such as the completeness and cardinality of independent sets of invariants for the bi-quadratics - a valuable property in the absence of apriori information of this type. 


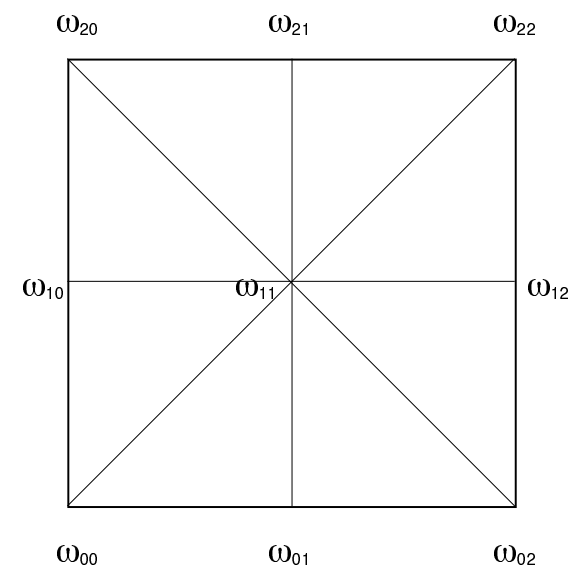

Fig. 1. Weight array for bi-quadratic patches

\section{Some applications}

\subsection{Patch equivalence}

Alternative techniques for constructing rational parametrisations of specific surfaces often produce parametrisations with identical vertex sets but different weight vectors. The invariants enable direct, efficient comparison of parametrisations to be performed (Corollary 2).

Example 1. Consider the Dupin cyclide defined, in the usual notation (see Foufou et al (2005)), by the parameters $a=6, c=2$ and $\mu=3$. The weights of the patch on the cyclide bounded by $\theta_{0}=\frac{2 \pi}{3}, \theta_{1}=\frac{4 \pi}{3}, \phi_{0}=\frac{5 \pi}{6}, \phi_{1}=\frac{3 \pi}{2}$ are shown, by the author's construction (Bez, 2007), to be:

$$
\omega=\left(6-\frac{\sqrt{3}}{2}, 3-\frac{\sqrt{3}}{2}, 6,3-\sqrt{3}, \frac{3}{2}-\sqrt{3}, 3,6-\frac{\sqrt{3}}{2}, 3-\frac{\sqrt{3}}{2}, 6\right)
$$

and by the methods of Foufou et al to be:

$$
\omega^{*}=(1,0.384,1,0.247,-0.042,0.5,1,0.384,1) .
$$

Clearly the sign patterns of $\omega$ and $\omega^{*}$ are the same and it is easy to show that $\varpi_{i}(\omega)=\varpi_{i}\left(\omega^{*}\right)$ for $0 \leq i \leq 6$. From Corollary 2 the parametrisations are equivalent.

\subsection{Standard parametrisations}

The transformations (11) enable bi-quadratic patches with positive weights to be re-parametrised with 3 weights taking the value +1 . If some negative 
weights occur, the signs are retained and re-parametrisations having 3 weights of absolute value 1 can be constructed. The constraints imposed by (11) do not permit all combinations of weight triples to be transformed to \pm 1 - for example the triple $\omega_{00}, \omega_{11}, \omega_{22}$ is impossible.

Assuming $\omega_{00}>0, \omega_{02}>0$ and $\omega_{20}>0$, a matrix, $N$, given by:

$N=\operatorname{diag}\left(\frac{1}{\omega_{00}}, \frac{1}{\left(\omega_{00} \omega_{02}\right)^{\frac{1}{2}}}, \frac{1}{\omega_{02}}, \frac{1}{\left(\omega_{00} \omega_{20}\right)^{\frac{1}{2}}}, \frac{1}{\left(\omega_{02} \omega_{20}\right)^{1 / 2}}, \frac{1}{\omega_{02}}\left(\frac{\omega_{00}}{\omega_{20}}\right)^{\frac{1}{2}}, \frac{1}{\omega_{20}}, \frac{1}{\omega_{20}}\left(\frac{\omega_{00}}{\omega_{02}}\right)^{\frac{1}{2}}, \frac{\omega_{00}}{\omega_{02} \omega_{20}}\right)$.

may be constructed, from (11), to determine 'normalizations' with $\omega_{00}=\omega_{02}=$ $\omega_{20}=1$. Signs may be adjusted, as required if $\omega_{00}>0, \omega_{02}>0$ and $\omega_{20}>0$ is not true; e.g., if $\omega_{02}<0$ then $\omega_{02}$ should be replaced by $\left|\omega_{02}\right|$ in $N$.

Alternatively, (11) permits weights $\omega_{00}, \omega_{01}$ and $\omega_{22}$ be transformed to unit modulus. Choosing $\omega_{00}$ to transform to 1 , the four distinct cases:

$$
\left(1, \pm 1, \omega_{02}^{*}, \omega_{10}^{*}, \omega_{11}^{*}, \omega_{12}^{*}, \omega_{20}^{*}, \omega_{21}^{*}, \pm 1\right)
$$

can occur - depending on the signs of $\omega_{01}$ and $\omega_{22}$. The matrix for these normalizations is:

$$
\operatorname{diag}\left(\frac{1}{\omega_{00}}, \frac{1}{\left|\omega_{01}\right|}, \frac{\omega_{00}}{\omega_{01}^{2}}, \frac{\left|\omega_{01}\right|}{\left|\omega_{22}\right|^{\frac{1}{2}} \omega_{00}^{\frac{3}{2}}}, \frac{1}{\omega_{00}^{\frac{1}{2}}\left|\omega_{22}\right|^{\frac{1}{2}}}, \frac{\omega_{00}^{\frac{1}{2}}}{\left|\omega_{01}\right|\left|\omega_{22}\right|^{\frac{1}{2}}}, \frac{\omega_{01}^{2}}{\omega_{00}^{2}\left|\omega_{22}\right|}, \frac{\left|\omega_{01}\right|}{\omega_{00}\left|\omega_{22}\right|}, \frac{1}{\left|\omega_{22}\right|}\right) .
$$

Example 2. Normalizing the author's weights (see Example 1 for patch details) to the form $\omega_{00}=\omega_{02}=\omega_{20}=1$ - using the matrix $N$ - we obtain, to three decimal places:

$$
(1,0.384,1,0.247,-0.042,0.5,1,0.384,1)
$$

which are identical to Foufou's weights for the patch at this precision - see Example 1.

\section{Concluding observations}

It is clear that the relationship between invariants and curves in the surface, demonstrated to exist in the bi-quadratic case by the canonical invariants derived in the paper, does not translate directly to Bézier surfaces of higher degree. For example in the bi-cubic case it would produce 16 invariants providing no scope for weight normalization. However it is well-known that, for surfaces of arbitrary degree, at least three weights may always be normalized to unit modulus by Möbius transformation - suggesting no more than $(n+1)^{2}-3$ invariants, of type $\varpi$, for surfaces of degree $(n, n)$ and $(n+1)(m+1)-3$ for degree $(m, n)$. These general cases and a number of related topics are under investigation by the author. 


\section{References}

Bez, H.E.(2007), Bounded domain, bi-quadratic rational parametrisations of Dupin cyclides, To appear in: International Journal of Computer Mathematics.

Farin, G. (1999), NURBS from Projective Geometry to Practical Use, Second Edition, A.K.Peters, 1999.

Foufou, S. Garnier, L. and Pratt, M.J. (2005), Conversion of Dupin cyclide patches into rational bi-quadratic Bézier form, Proceedings of the IMA Mathematics of Surfaces XI, Springer Verlag, Lecture Notes in Computer Science 3604, 201-218.

Olver, P.J. (1999), Classical Invariant Theory, London Mathematical Society Student Texts, 44, Cambridge University Press, 1999.

Patterson, R.R. (1985), Projective transformations of the parameter of a Bernstein-Bézier curve, ACM Trans. on Graphics 4(4), 276-290. 